\title{
Examining the energy loss in the Inverted Pendulum Model for
} rocking bodies

\author{
M. N. Chatzis ${ }^{1}$, M. García Espinosa ${ }^{2}$ and A. W. Smyth ${ }^{3}$
}

\section{Abstract}

5 Understanding of the mechanisms that may lead to failure of rocking bodies is of significant importance to quantifying and minimizing the associated risk. The most common model to describe the rocking problem is the Inverted Pendulum model $(I P M)$ proposed by Housner in 1963, which has been followed by multiple researchers. It is often claimed that the IPM is an acceptable model if behaviors such sliding, uplifting, deformability and the three-dimensional nature of the response can be restrained mechanically. However, even in a suitably chosen case where the previous may indeed have a minimal effect in the response, there are still uncertainties with regard to the assumption introduced in the IPM of how energy is lost during impacts. This paper deals with this assumption and investigates the effects in the stability of rocking bodies. The effect of this assumption is discussed showing that the associated uncertainty propagates to the conclusion of whether a rocking body would survive or fail when subjected to a ground excitation. A method that quantifies this uncertainty by making use of a dynamic property of the rocking system is introduced. 
Palmeri and Makris, 2008). Two models, depending on whether the base of the rocking body is flat or has concentrate supports the Winkler Model $(W M)$ and Concentrate Spring Model (CSM) respectively, that take into account all the previous phenomena were proposed in (Chatzis and Smyth, 2012b). A three dimensional model ignoring sliding, uplift and deformability was proposed in (Zulli et al., 2012) and the extensions of the $W M$ and $C S M$ into three dimensions were presented in (Chatzis and Smyth, 2012a). Those papers have highlighted that each of these assumptions is indeed of significant importance and should not be omitted. Additionally the stability investigations in (Chatzis and Smyth, 2012b,a) showed that neglecting sliding and the three-dimensional nature of the response are not necessarily conservative assumptions.

It is often however claimed that the effect of these omitted phenomena can be minimized mechanically. For example, sliding may be minimized using a high friction material between the body and the base, while the effects of deformability are substantially smaller for relatively stiff materials for the body and the support medium, the chance of the body being in a free flight mode is reduced as it becomes more slender and assuming that all the excitations are horizontal, while the out of plane response of the body can be minimized if the perpendicular dimension of the base of the body, the length, is substantially greater to the dimension of the in plane dimension of the base, the width. Nevertheless, even if the effect of the previous phenomena is minimized, the IPM as introduced by (Housner, 1963), makes an explicit assumption about the energy lost during impacts: the location of the vertical impulsive force generated during impact is assumed to be at the future rocking corner. Therefore, the angular momentum is conserved about this point.

Existing works in the literature have used alternative assumptions about the loss of energy during impacts (Zhang et al., 2012; Yim et al., 1980; Dimitrakopoulos and DeJong, 2012; Voyagaki et al., 2014; ElGawady et al., 2011). These are usually expressed through the use of alternative values for the reduction of energy factor. While some of those works have attributed the use of different reduction factors to the effect of the ignored phenomena, i.e., sliding, uplift, deformability and the three-dimensional nature of the response, it should be noted that their inclusion would result in a substantially different dynamic behavior including dynamic patterns that could not be predicted by the IPM for any assumed value of the reduction of energy factor. This work will illustrate that conserving momentum with respect to the future rocking corner is not the only admissible solution to the problem even if, the examined body is such so that the ignored phenomena have indeed a minimal effect on its response.

It will be shown in this work that the vertical impulse during an impact may be applied in any position between the future rocking corner and the midpoint of the base, without violating the rest of the assumptions. This position can, hence, be considered as a random variable with a related uncertainty. This paper illustrates of how this uncertainty propagates to the stability of a rocking body subjected to a ground excitation.

Stability diagrams are developed for a rigid body subjected to ground excitations of the form of single, double or triple sinusoidal, cosinoidal or square pulses. 
Conclusions are drawn on the effect of the position of the vertical impulse on the stability of the body. To quantify the propagation of the uncertainty on the stability of the body, a probabilistic approach is developed using the dynamic properties of the stability of a rocking body. The approach is initially formulated for the stability of rocking bodies subjected to single cycloidal pulses as in (Zhang and Makris, 2001) and then extended to the case of multiple pulses (Plaut et al., 1996). Finally, the effect of the occurring uncertainty is demonstrated by generating fragility curves for earthquake inputs generated with a stochastic method (Shinozuka and Deodatis, 1991).

\section{Model and Assumptions}

\section{Dynamic Model}

This section provides a brief description of the IPM (Housner, 1963). The rocking body and the properties used in the following equations are defined in Figure 1. In this paper the convention which is used for the axes is shown in Figure 1, with $x$ and $y$ being the horizontal and vertical axis respectively, and $\theta$ the rotation. The block can be fully defined by its mass $m$, its moment of inertia $I_{0}$ about one of the two corners 0 and $0^{\prime}$, while $b$ and $h$ are the half-width and half-height of the body, respectively. Furthermore, $R$ is the distance from a corner to the center of mass, $R=\sqrt{h^{2}+b^{2}}$. The slenderness of the block is defined by the angle $\alpha=\arctan \frac{b}{h}$. Depending on the sign of $\theta$ the body rocks with respect to the corresponding corner, and hence can be simulated by a pendulum whose pin is located at that corner as shown in Figure 1. This of course implies, that the body cannot slide, or experience a free-flight mode, that both the body and the ground are rigid, and that the response of the body is strictly planar, which are assumptions that will be adopted in this paper.

[Figure 1 about here.]

The motion of the body is defined through three patterns. Patterns A and $\mathrm{B}$ are the pendulum patterns corresponding to $\theta<0$ and $\theta>0$ respectively, while pattern $\mathrm{C}$ is the static pattern $\theta=0$ and $\dot{\theta}=0$, i.e., when the body is at rest. The block is subjected to a positive horizontal, $\ddot{x}_{g}$, and vertical, $\ddot{y}_{g}$, ground accelerations. $F_{x}$ and $F_{y}$ are D'Alembert fictitious forces defined by $F_{x}=-m \ddot{x}_{g}$ and $F_{y}=-m \ddot{y}_{g}$. The ground is experiencing only translational accelerations and not rotational as for example examined in Virgin et al. (1996). If moments are taken about the center of rotation 0 or $0^{\prime}$, the equations of motion, using Newton's second law, are respectively:

$$
\begin{gathered}
\ddot{\theta}=-p^{2} \sin (\alpha-\theta)+\frac{m \ddot{x}_{g}}{I_{0}} R \cos (\alpha-\theta)-\frac{m \ddot{y}_{g}}{I_{0}} R \sin (\alpha-\theta) \text { for } \theta>0 \\
\ddot{\theta}=p^{2} \sin (\alpha+\theta)+\frac{m \ddot{x}_{g}}{I_{0}} R \cos (\alpha+\theta)+\frac{m \ddot{y}_{g}}{I_{0}} R \sin (\alpha+\theta) \text { for } \theta<0
\end{gathered}
$$


where $p^{2}=m g R / I_{0}$.

In the static pattern the ground applies to the body horizontal and vertical reactions, $f_{x}$ and $f_{y}$ respectively, so that the static equilibrium is retained. The vertical reaction $f_{y}$ is applied at a distance $d$ measured, by convention, from the right corner 0 . Since it is assumed that the ground cannot provide any tensile forces, the position of the vertical reaction force is bounded between the two corners, hence: $0 \leq d \leq 2 b$. If in order to retain static equilibrium the obtained value of $d$ is outside those limits, then the static equilibrium cannot be retained as it is assumed that the ground interface is unable to provide tensile forces. Hence, as $d=0$ or $d=2 b$ the body transitions from the static pattern to patterns $B$ or $A$, respectively.

In order to switch between patterns $A$ and $B$, an impact occurs as is shown in Figure 2. This transition takes place when $\theta=0$. During this impact, the body will experience impulsive forces. The corresponding resultants of the horizontal and vertical impulses are defined as $J_{x}$ and $J_{y}$ respectively.

[Figure 2 about here.]

The assumption of conserving momentum with respect to the future rocking corner during impacts, is equivalent to assuming that the vertical impulse acts on that corner. To obtain $J_{y}$ a distribution of vertical impulses along the base of the body has to be assumed. The restrictions for that distribution are that the distributed impulses are applied at the contact interface, i.e. between the two corners, and that they correspond to compressive impulsive forces. For the purposes of illustration of the following Figure 3 and without any loss of generality, a flat base is assumed for the body and hence the distributed impulses can be applied at any point between the two corners.

Figure 3 represents one of the infinitely many admissible distributions that could be considered for the distributed vertical impulses. Since the body is rigid the distribution can be completely represented by the resultant impulse $J_{y}$ which is located at the centroid of the distribution. This centroid, without any loss of generality, may be at a distance $\lambda b$ measured from the post-impact rocking corner towards the pre-impact rocking corner. The exact geometry of the base plays an important role in the response of the body as has been shown in (Chatzis and Smyth, 2012b; Chatterjee and Ruina, 1998). A nonflat multi-contact base would result into a non-continuous impulse distribution along the base. Nonetheless, this does not influence any of the results of the paper as the continuous nature of the distribution of the impulses is not made use of. For Figure 3 the pre- and post-impact rocking corners are assumed to be $0^{\prime}$ and 0 respectively. The requirement for all forces from the interface to be compressive, including impulsive forces and the corresponding impulses result in the following constraints: $J_{y}>0$ and $0 \leq \lambda \leq 2$. The latter constraint translates to the centroid being between the two corner points.

[Figure 3 about here.] 
Formulating the momentum equations linking the time instances after and before the impact as in Figure 2, the following three momentum equations are obtained:

$$
\begin{aligned}
m \dot{x}^{+} & =m \dot{x}^{-}+J_{x} \\
m \dot{y}^{+} & =m \dot{y}^{-}+J_{y} \\
I_{c} \dot{\theta}^{+} & =I_{c} \dot{\theta}^{-}-(b-\lambda b) J_{y} \operatorname{sign}\left(\dot{\theta}^{-}\right)+h J_{x}
\end{aligned}
$$

where $\dot{x}^{+}, \dot{y}^{+}, \dot{\theta}^{+}$and $\dot{x}^{-}, \dot{y}^{-}, \dot{\theta}^{-}$are: the horizontal, vertical and angular velocity after and before impact and $I_{c}$ is the moment of inertia about the center of the block. The sign in equation (2) accounts for whether the future rocking corner is 0 or $0^{\prime}$, with $\operatorname{sign}\left(\dot{\theta}^{-}\right)$being + or - respectively. Due to the pendulum kinematic constraints:

$$
\begin{array}{ll}
\dot{x}^{+}=-h \dot{\theta}^{+} & \dot{y}^{+}=b\left\|\dot{\theta}^{+}\right\| \\
\dot{x}^{-}=-h \dot{\theta}^{-} & \dot{y}^{-}=-b\left\|\dot{\theta}^{-}\right\|
\end{array}
$$

Thus, the unknowns of the system are $\dot{\theta}^{+}, J_{y}, J_{x}$ and $\lambda$. To find a solution of the system, it is necessary to assume the value of one of the unknowns, for example, $\lambda$. While this is often assumed to be equal to zero in the literature, it is important to note that there would not be any violation of the momentum equations assuming any value for $\lambda$. But if one takes into account the previous discussion, any value of $\lambda \in[0,2]$ could be used. The angular velocity after impact is defined through the reduction of energy factor, $r$, which relates the kinetic energy before and after impact.

$$
r=\frac{\left(\dot{\theta}^{+}\right)^{2}}{\left(\dot{\theta}^{-}\right)^{2}}=\left(\frac{1-\frac{3}{4}(2-\lambda) \sin ^{2} \alpha}{1-\frac{3}{4} \lambda \sin ^{2} \alpha}\right)^{2}
$$

Leading to the equation: $\dot{\theta}^{+}=\sqrt{r} \dot{\theta}^{-}$. It can be seen from equation (5) that if $\lambda>1 \Rightarrow r>1$, hence energy would be generated during the impact, which is not physically possible in this problem where the ground experiences only translational accelerations. Thus, two material assumptions, the lack of tensile forces from the interface and that there cannot be an increase of the energy during an impact, result in $\lambda$ taking admissible values in the interval $[0,1]$. Any value of $\lambda \in[0,1]$ is completely compatible with the assumptions made in the $I P M$. To this point it should be noted that, researchers have suggested the use of values of smaller reduction of energy factors than the one suggested by Housner in (Housner, 1963), or equivalently have suggested negative values for $\lambda$, to account for additional loss of energy during impact, potentially due to the ignored sliding, bouncing, deformability of the ground, or the three dimensional nature of the response. However, if those assumptions were to be violated, the 
dynamic response of the body would include additional dynamic patterns, whose effect on the response cannot be approximated by an IPM with any value of $\lambda$. In other words, one should either use a model capable of taking into account those phenomena, or if these phenomena can be neglected then an IPM can be used assuming that $\lambda \in[0,1]$. This work will assume the latter.

Finally, it should be noted that the occurring $\sqrt{r}=\frac{1-\frac{3}{4}(2-\lambda) \sin ^{2} \alpha}{1-\frac{3}{4} \lambda \sin ^{2} \alpha}$ should be positive. Negative solutions do not make physical sense and they would result in a free-flight pattern that is incompatible with the IPM. This paper will deal with bodies of aspect ratio $h / b$ so that $\sqrt{r}$ is positive even for $\lambda=0$. As algebraically $\sqrt{r}$ becomes larger for larger values of $\lambda$, this guarantees that $\sqrt{r}>0$ for any of the bodies and values of $\lambda$ examined in this paper.

\section{Numerical Simulation}

[Figure 4 about here.]

To integrate this model over time, the dynamic equations in (1) are brought to state-space form and are integrated over time using a variable time stepping Runge-Kutta 4-5 pair as presented in (Dormand and Prince, 1980). This algorithm uses a $4^{\text {th }}$ order Runge-Kutta to propagate the solution over time and performs a comparison versus the prediction obtained from the $5^{\text {th }}$ order Runge-Kutta estimating an error from the difference of the two methods. The time step is varied in order to retain the estimated relative and absolute error within some bounds.

Unlike typical equations of motion, the ones describing the behavior of the IPM are not smooth, i.e., infinitely differentiable as there is a switch of the statespace equations from one pattern to the other. Additionally, the velocity state $\dot{\theta}$ is discontinuous around the time instance of an impact. In order to switch from one pattern to the other an event driven function algorithm is suggested in (Shampine and Gordon, 1975), that allows for stopping the integration around a specific time instance at which an event is located, is defined. In this problem the events are the transitions between patterns or the detection of toppling, i.e., failure, of the body, i.e., $\|\theta\|=\pi / 2$. Those are located online by the algorithm as implemented in Matlab's ode45 solver with the Events option Mathworks (n.d.). Each time a transition between patterns is located, the algorithm stops the integration and switches the state-space equations used to account for the new pattern. Furthermore, if the detected event was an impact then the velocity of the body is modified according to equation (5) to account for the loss of energy during the impact.

Equation (5) implies that eventually $\dot{\theta}$ tends to zero but never becomes numerically equal to zero. In other words, in theory the body can never go back to the static pattern from one of the two pendulum patterns. This however, is impractical in terms of the time integration and may lead to numerical instabilities when equation (5) is applied to values of the pre-impact velocity, or equivalently the kinetic energy just before the impact $E_{k}$, close to the machine tolerance. To avoid that and ensure stability and robustness of the algorithm, a 
condition is imposed after an impact event regarding the minimum value of the kinetic energy of the body: if the energy of a body after impact is smaller than this minimum value, then the static pattern is enforced as shown in Figure 4. The integration continues until a new switch is located, or until the stopping criteria for integration are met. These stopping criteria are either that toppling of the body was detected, or that the upper bound of the requested time window of integration was met, i.e., the body survived this excitation. Figure 4 shows the numerical implementation.

\section{Deterministic effect of $\lambda$ on the stability of the body}

As stated in the previous section (Model and Assumptions), $\lambda$ has an associated uncertainty. The source of this uncertainty is the lack of modeling the contact physics in the IPM, which allows for an infinite number of admissible distributions for the vertical impulses. This can be thought of as a 'structural' uncertainty of the IPM. This section will examine how different values of $\lambda$ would affect the stability of a rocking body when subjected to a ground acceleration $\ddot{u}$. In this first section a single cycloidal pulse stability analysis will be conducted, similar to the one presented in (Zhang and Makris, 2001). A free standing block with $\mathrm{p}=2.1401$ and $\alpha=0.2501$ as in (Zhang and Makris, 2001), as shown in Figure 1, is subjected to single cycle sinusoidal, cosinoidal and square pulses for a range of angular frequencies $\omega_{u}$ and amplitude $u_{0}$

$$
\begin{array}{ll}
\ddot{u}=0, & \text { if } \mathrm{t}>\mathrm{T} \\
\ddot{u}=u_{0} \mathbf{H}\left(\omega_{u} t\right), & \text { if } 0 \leq \mathrm{t} \leq \mathrm{T}
\end{array}
$$

where $T=\frac{2 \pi}{\omega_{u}}, \mathbf{H}$ is either of the functions sin, cos, square for the sinusoidal, cosinoidal and square pulses respectively, with the square function being defined as the following periodic function with period $T$ :

$$
\begin{array}{ll}
\operatorname{square}\left(\omega_{u} t\right)=+1, & \text { if } \quad 0<t<T / 2 \\
\operatorname{square}\left(\omega_{u} t\right)=-1, & \text { if } \quad T / 2<t<T
\end{array}
$$

As noted in (Zhang and Makris, 2001), three areas can be observed in a stability diagram of a body subjected to single cycloidal pulses: survival, failure after a single impact and failure without impact. The term 'impact' in this paper refers to the impact of the body with the ground. Stability diagrams are generated in Figure 5(a) showing these areas for a range of combinations of $u_{0}$ and $\omega_{u}$ for a sinusoidal pulse. Note that there are no failures for $\frac{u_{0}}{\tan (\alpha) g}<1$, because this is the critical value to initiate rocking. The non-linearity of the problem is apparent by the fact that there are pulses of constant frequency, for which as the amplitude of the pulse, $\frac{u_{0}}{\alpha g}$, is increased the block goes from the 
stable region to the unstable (failure with impact), re-transitions to the stable region and has a final transition to the unstable region (failure without impact).

[Figure 5 about here.]

Thanks to the similarity property described in (Zhang and Makris, 2001; Dimitrakopoulos and DeJong, 2012) the results for the diagram are applicable to bodies which have the same aspect ratio but potentially different size, as expressed through $R$, as long as the same value of $\omega_{u} / p$ is examined. It should further be noted that, as indicated in this paper, an additional parameter required for ensuring similarity between two such bodies is the assumption of the same value of $\lambda$ for both of them. In (Zhang and Makris, 2001), it was assumed that the vertical impulse during an impact is applied at the future rocking corner, i.e., $\lambda=0$. However, as discussed in section Model and Assumptions, $\lambda$ can attain values between zero and one. Figure 5 shows the stability diagrams for all three types of pulses for values of $\lambda=0$ and $\lambda=1$. A significant increase of the failure with impact can be observed in all three cases, as $\lambda$ goes from zero to one. This is reasonable as larger values of $\lambda$ correspond to a smaller loss of energy during impacts and specifically the extreme case of $\lambda=1$ case corresponds to conservation of energy during impacts. For the cosinoidal pulse, as can be seen in Figures 5(b) and 5(e), the single impact instability region is smaller than in Figure 5(a) and 5(d) and the boundary between non-impact and safety regions is steeper. On the other hand the square pulse, presents similarities to the sinusoidal pulse as can be seen in Figure 5(c) and 5(f).

[Figure 6 about here.]

As expected, the area of failure without impact is unaffected by $\lambda$. This behaviour can be observed for any aspect ratio $h / b$ and for the purpose of illustrating this, two different aspect ratios are used in Figure 6 for all three pulses. Figure 6 represents the boundaries between survival and failure for different vales of $\lambda$. As the assumed value of $\lambda$ is increased the area of failure with impact is expanding by including more pairs of $u_{0}$ and $\omega_{u}$. This significant behaviour means that, for a given pair of values $\omega_{u}$ and $u_{0}$ in the area defined between the boundary lines for the areas of failure with impact for $\lambda=0$ and $\lambda=1$, a body which fails for a given value of $\lambda$ will also fail for higher values of $\lambda$. Hence, for the case of single cycle pulses, there is a value of $\lambda=\lambda^{*}$, such that a point on the stability diagram belonging to the predefined region will fail for any $\lambda>\lambda^{*}$ and survive for $\lambda<\lambda^{*}$ :

$$
\begin{aligned}
& \text { if } \lambda<\lambda^{*} \rightarrow \text { SURVIVAL } \\
& \text { if } \lambda \geq \lambda^{*} \rightarrow \text { FAILURE }
\end{aligned}
$$

It should also be observed that points in the diagram that fail for $\lambda=0$ fail for any of the allowable values of $\lambda$. Notice that the survival or failure of the remaining points on the stability diagram, outside the area defined earlier, does not depend on the value of $\lambda$. 
Following the property defined in equation (8), the bisection method is applied to calculate $\lambda^{*}$, i.e., the value of $\lambda$ signifying the transition between failure and survival. The bisection method detects this transition with the precision of a tolerance imposed (tolerance $=10^{-3}$ ). Figure 7 shows the corresponding values of $\lambda^{*}$ for a single sinusoidal, cosinoidal and square pulse. It can be observed that the mapping of $\lambda^{*}$ to an $\left(\omega_{u}, u_{0}\right)$ pair depends on the type of the input.

[Figure 7 about here.]

Figure 8 shows a comparison between the three Pulses for the cases of $\lambda=0$ and $\lambda=1$. Notably, the square pulse results in a higher range of $u_{0}$ and $\omega_{u}$ values that signify failure. On the other hand, the cosinoidal pulse seems to have a usually safer behaviour than the sinusoidal and square pulses. However, there are areas on the diagram for which any of the three inputs may result in failure and the other two to safety. For example, the cosinoidal pulse fails for the case of $\lambda=0, \omega_{u}=4 p$ and $u_{0}=3.7 \alpha \mathrm{g}$, while the square pulse survives. Comparing the cosinoidal and the sinusoidal pulses for the case of $\lambda=0$ for $\omega_{u}=4 p$ and $u_{0}=5.9 \alpha \mathrm{g}$, the former fails but not the latter. In general, for a specific value of $\omega_{u}, u_{0}$ and $\lambda$, due to the non-linearity of the problem, there will be areas where the survival or failure of the body will depend on the type of the input.

[Figure 8 about here.]

At this point, it can be observed that variations of the value of $\lambda$ significantly change the failure with impact areas. In the literature, it is claimed that $\lambda=0$, hence according to equation 5 the smallest allowable value of $r$ is used. However, this paper has already demonstrated that the body has a greater tendency to collapse as the value of $\lambda$ is increased. Hence, assuming that $\lambda=0$, is a nonconservative assumption. It should further be noted that in Figure 5(a) when $\omega_{u} / p$ is small, the minimum overturning acceleration for failure is given by the lower limit of the failure with impact area. As the frequency of the input pulse is increased, this area disappears and hence the minimum amplitude for failure is given by the boundary of the failure with no impact area. Hence at some frequency, there is a jump of the minimum acceleration for overturning. This is a favorable property as the required amplitude for overturning the body for an input beyond that transition frequency is substantially larger. Nevertheless, the value of this transition frequency increases as $\lambda$ is increased, as shown in Figure 6 , reducing the range of these favorable, in terms of the required amplitude of overturning, frequencies.

For the remainder of this work, the following dimensions will be adopted $p=2.1401$ and $\alpha=0.3218$ which correspond to $\mathrm{h}=1.5240 \mathrm{~m}, \mathrm{~b}=0.5080 \mathrm{~m}$ and $h / b=3$. 


\section{Quantifying the Probability of Failure.}

\section{Probabilistic models for the effect of $\lambda$ on the stability of the body.}

Based on the previous discussion in section Model and Assumptions, $\lambda$ is a random variable between zero and one and hence it is associated with some probability of occurrence. As a result, the outcome of a body being subjected to a single cycloidal pulse of a specific $u_{0}$ and $\omega_{u}$ is also linked with a corresponding probability of survival/failure. While, there are points in the stability diagram, i.e., pairs of values $\left(\omega_{u}, u_{0}\right)$, that fail or survive for any of the possible values of $\lambda$, there are also points for which, the deterministic property defined on equation (8) correspond to a probability property: the probability of the body failing or surviving is only the probability of $\lambda$ being smaller or greater than $\lambda^{*}$ :

$$
\begin{aligned}
& P\left(\lambda>\lambda^{*}\right)=\text { PROBABILITY OF FAILURE } \\
& P\left(\lambda<\lambda^{*}\right)=\text { PROBABILITY OF SURVIVAL }
\end{aligned}
$$

In this work, two types of distributions are used for modelling $\lambda$ : uniform and truncated Gaussian distributions. The latter is defined in terms of the mean, $\mu$, and the standard deviation, $\sigma$. Assuming a value of $\mu$ closer to a value implies that this is expected as the most probable value of $\lambda$, while $\sigma$ quantifies the dispersion of a set of values. Thus a $\sigma$ closer to zero indicates that the expected values of $\lambda$ are closer to the mean. An increase of the value of sigma indicates that there is smaller certainty about the expected value of $\lambda$. The limits of $\mu$ and $\sigma$, for a truncated Gaussian distribution, are $\mu_{u}=0.5$ and $\sigma_{u}=$ 0.5 , respectively. These values correspond to the uniform distribution, which corresponds to the assumption that any value of lambda between zero and one is equally possible. Equations 10 and 11 refer to the Probability Density Function $(P D F), P D F_{U}(x)$, and Cumulative Density Function $(C D F), C D F_{U}(x)$, of the Uniform Distribution.

$$
\begin{gathered}
P D F_{U}(x)= \begin{cases}1 & \text { for } 0 \leq x \leq 1, \\
0 & \text { for } x<0 \text { or } x>1\end{cases} \\
C D F_{U}(x)= \begin{cases}0 & \text { for } x<0, \\
x & \text { for } x \in[0,1), \\
1 & \text { for } x \geq 1\end{cases}
\end{gathered}
$$

It is often convenient to define a truncated Gaussian in terms of an untruncated Gaussian of $\mu_{u}$ and $\sigma_{u}$. The truncated Gaussian distribution is defined as follow:

$$
P D F_{T}\left(x ; \mu_{u}, \sigma_{u}, 0,1\right)=\frac{\phi(\xi)}{\Phi(\beta)-\Phi(\alpha)}
$$




$$
\begin{gathered}
C D F_{T}\left(x ; \mu_{u}, \sigma_{u}, 0,1\right)=\frac{\Phi(\xi)-\Phi(\alpha)}{\Phi(\beta)-\Phi(\alpha)} \\
\mu=\mu_{u}+\frac{\phi(\alpha)-\phi(\beta)}{\Phi(\alpha)-\Phi(\beta)} \sigma_{u} \\
\sigma=\sigma_{u}^{2}\left(1+\frac{\alpha \phi(\alpha)-\beta \phi(\beta)}{\Phi(\alpha)-\Phi(\beta)}-\left(\frac{\phi(\alpha)-\phi(\beta)}{\Phi(\alpha)-\Phi(\beta)}\right)^{2}\right)
\end{gathered}
$$

where $\mu_{u}$ and $\sigma_{u}$ are the values of the untruncated Gaussian distribution, $\xi=$ $\frac{x-\mu_{u}}{\sigma_{u}}, \alpha=\frac{0-\mu_{u}}{\sigma_{u}}$ and $\beta=\frac{1-\mu_{u}}{\sigma_{u}},[0,1]$ are the limited values $[0,1], \phi$ and $\Phi$ are the PDF the $C D F$ of the normal Gaussian distribution $(\mu=0$ and $\sigma=1)$, respectively.

The probability of failure of the body for a range of values of $u_{0}$ and $\omega_{u}$ should then be calculated. Monte Carlo simulations can be used to calculate the probability of failure. A number of generated random samples of $\lambda$ are used to calculate the probability of failure for each value of $u_{0}$ and $\omega_{u}$ for a given distribution. The number of generated samples of $\lambda$ is increased until convergence is achieved. For the case of the uniform distribution, the random values are modelled using the 'rand' tool from Matlab which generates uniformly distributed random numbers. For the truncated Gaussian distribution, the algorithm described in Robert (1995) is used to generate samples. The probability of failure is calculated for a pair of $u_{0}$ and $\omega_{u}$, as the sum of all observed failures divided by the number of samples of $\lambda$.

Alternatively, the probability of survival or failure can be defined directly using the property defined in equation 9. In this approach, having defined the values of $\lambda^{*}$, for a body of a given aspect ratio and type of input, one can then apply equation 9 for each pair of points $\left(u_{0}\right.$ and $\left.\omega_{0}\right)$ to generate the corresponding probability of survival/failure in the area of points for which equations 8 and 9 are applicable.

The advantage of the second approach is that the second step, i.e., the transition from the $\lambda^{*}$ mapping to the probability of failure does not require any further dynamic simulations, but only the calculation of the associated probabilities, that solely depend on the assumed distributions and the values of $\lambda^{*}$ corresponding to each point. This probability can, for the cases examined, be calculated analytically. This results in an overall substantial reduction of calculation cost in comparison to the Monte Carlo approach. It should be noted that the Monte Carlo simulations mentioned in this paper use 1000 samples.

For any single cycloidal pulse input, it can be observed as for example in Figure 5, that the area of failure without impact does not change for different values of $\lambda$. Hence, the probability of failure in these areas is $100 \%$. Because $\lambda$ cannot be greater than one, the area that corresponds to survival for the case of $\lambda=1$ has a probability of failure of $0 \%$. Hence, as in previous sections, only the points for which equations (8) and (9) are applicable, are analysed. Figure 9 compares the Monte Carlo Simulations versus the defined probabilistic 
approach for a single cosinoidal pulse. It can be seen that there is an excellent match between the two methods. In fact the difference between the two methods is controlled by the number of samples used for the Monte Carlo method and the error tolerance chosen for numerically estimating equation (9). By increasing the former and reducing the latter a desired difference tolerance between the two methods can be achieved. The same can be verified regardless of the input, dimensions of the body or the distribution assumed for $\lambda$.

[Figure 9 about here.]

\section{Effect of the Uncertainty of $\lambda$ in the estimate of the out- come.}

The probability of failure of the body for a single cosinoidal pulse input, assuming truncated Gaussian distributions of $\lambda$ corresponding to different values of $\mu$ and $\sigma$ are compared in Figure 10. Increasing $\mu$ and keeping $\sigma$ constant as in Figures 10(a) and 10(c) or 10(b) and 10(d), the area of points with probability of failure close to $100 \%$ increases. This is in agreement with what was presented in section Deterministic effect of $\lambda$ on the stability of the body, as higher values of $\mu$ correspond to a higher expected value of $\lambda$. On the other hand, increasing $\sigma$ and keeping $\mu$ constant, as in Figures 10(a) and 10(b) or 10(c) and 10(d), results in reducing the size of areas with an almost constant probability of failure.

In Figure 10 there are points whose probability of failure is very close to $50 \%$. A probability of failure of $50 \%$ means that there is as much certainty about the outcome using the mode, as that which would occur by flipping a coin. Thus, there is a very large uncertainty to the model's prediction on whether the body is expect to survive or fail. The same practically holds for a range of probabilities close to $50 \%$, e.g. $40-60 \%$. Hence points in the diagram with probabilities of failure in that range should be further examined.

[Figure 10 about here.]

In attempting to understand the effect of the assumed distribution of $\lambda$ on the region of points with the aforementioned range of probabilities of failure, the CDFs for assumed Truncated Gaussian distributions of $\lambda$ occuring from different values of $\mu$ and $\sigma$ are illustrated in Figure 11(a). The vertical axis represents the the probability of $\lambda$ being greater than the value indicated in the horizontal axis. Those distributions are used in the stability analysis of a body with $\alpha=0.2501$ and $p=0.3218$ subjected to a single cycloidal pulse ground excitation with the results shown in 11(b). A point in the region of the stability diagram that satisfies the property of equation 9 would have a probability of failure of $X \%$, if $1-C D F\left(\lambda^{*}\right)=X \%$. For the reasons explained earlier it is hence beneficial to define in Figure 11(a), the values of $\lambda^{*}$ that would correspond to a probability of failure of $40 \%$ and $60 \%$, the corresponding values of $\lambda^{*}$ will be denoted as $\lambda_{60}^{*}$ and $\lambda_{40}^{*}$, respectively.

In Figure 11(a) the values of $\lambda_{40}^{*}$ and $\lambda_{60}^{*}$ for each distribution are selected. The values are shown in Table 1. In Figure 11(b), the points which correspond 
to $\lambda^{*}$ values equal to $\lambda_{60}^{*}$ and $\lambda_{40}^{*}$ are represented with lines. These lines are also the boundaries of the points of this uncertain region between $40 \%$ and $60 \%$ probability of failure for each distribution. The dashed line represents the $60 \%$ boundaries and the continuous line the $40 \%$ boundary. In Table 1 , for $\mu=0.2$, the $\lambda_{60}^{*}$ value decreases as $\sigma$ is increased. However, for $\lambda_{40}^{*}$, the decrease is smaller. From Figure 7(b), it can be observed that the values of $\lambda^{*}$ decrease when moving towards the bottom left corner, and increase while moving towards the upper right corner. The same behaviour is observed in the Figure 11(b). The $60 \%$ line moves towards the bottom left corner as $\sigma$ is increased and is almost constant for the $40 \%$ line. Hence, the region of the points with probabilities between $40 \%$ and $60 \%$ expands towards the bottom left corner. In Table 1, for $\mu=0.8, \lambda_{60}^{*}$ is almost constant whereas $\lambda_{40}^{*}$ increases as $\sigma$ is increased. In Figure 11(b), the uncertain region extends towards the upper right corner as $\sigma$ is increased. In Table 1 , for values of $\mu=0.5$, the probability of $\lambda^{*}$ increases and decreases symmetrically as the uniform distribution is approached, matching with what is observed in Figure 11(b) where the uncertain region expands in both directions as $\sigma$ is increased.

It was shown in the previous paragraph, how one can determine the behaviour of this uncertain region of points with probabilities between $40 \%$ and $60 \%$, using only the $\lambda^{*}$ for a specific body and excitation and the CDF for the assumed distribution of $\lambda$.

[Table 1 about here.]

[Figure 11 about here.]

\section{Stability Analysis: Multiple-Pulses}

This section is an extension of single pulses to multiple pulses. To this end, the body is subjected to double and triple cycloidal pulses, by modifying equation (6) to:

$$
\begin{array}{ll}
\ddot{u}=0, & \text { if } t>N_{c} T \\
\ddot{u}=u_{0} \mathbf{H}\left(\omega_{u} t\right), & \text { if } 0 \leq t \leq N_{c} T
\end{array}
$$

where $N_{c}$ is the number of cycles of the pulse. Figure 12 illustrates areas of failure with impact for different values of $\lambda$ subjected to double sinusoidal and cosinoidal pulses.

[Figure 12 about here.]

It should be noticed that not all points agree with the property described on (8). For example, in Figure 12(a) for a double sinusoidal pulse input some points fail for $\lambda=0$ while they do not for greater values of $\lambda$. And in general, for a pair of $u_{0}$ and $\omega_{u}$, there are points for which the body fails for a value of $\lambda, \lambda_{2}$, and survives for another value of $\lambda, \lambda_{1}$, even when $\lambda_{1}>\lambda_{2}$. The 
same phenomenon can be observed in Figure 12(b) for a double cosinoidal pulse input. For $\omega_{u}=3.5 p$ and $u_{0}=5 \alpha g, \lambda=0$ the body fails, whereas for $\lambda=0.2$ it survives. Hence, for each $\omega_{u}$ and $u_{0}$, there might be multiple transitions from survival to failure as the value of $\lambda$ increases. This is illustrated in Figure 13. Those transitions essentially correspond to multiple roots in accordance to what was defined for single pulses in section .

Due to equation (8) not being applicable for this case, two methods are investigated for determining the probability of failure of the body: $i$ ) a multiroot finding method ii) Monte Carlo Simulations. The latter is practically the same as explained in section Probabilistic models for the effect of $\lambda$ on the stability of the body, where the probability of failure for a point on the stability diagram is calculated as the sum of all the occurrences of failure over the total number of samples of $\lambda$ used.

[Figure 13 about here.]

A multiroot finding method is used based on a modification of what was presented in for single pulses. This method determines for a pair of $\omega_{u}$ and $u_{0}$ all the corresponding roots, i.e., values of $\lambda, \lambda^{*}$, for which there is a transition from survival (S) to failure (F) and vice-versa, as shown in Figure 13. Once all roots for a pair of values of $\omega_{u}$ and $u_{0}$ are found, the probability of survival or failure is determined depending on whether the body fails or survives for $\lambda=0$ : Following Figure 13, if the body fails for $\lambda=0$, the probability of survivals is defined as in equation (17). Otherwise, the probability of survival is defined as in equation (18).

$$
\begin{aligned}
P_{S} & =P\left(\left(0<\lambda<\lambda_{1}^{*}\right) \cup\left(\lambda_{2}^{*}<\lambda<\lambda_{3}^{*}\right) \cup \cdots\right) \\
& =P\left(0<\lambda<\lambda_{1}^{*}\right)+P\left(\lambda_{2}^{*}<\lambda<\lambda_{3}^{*}\right) \cdots \\
P_{S} & =P\left(\left(\lambda_{1}^{*}<\lambda<\lambda_{2}^{*}\right) \cup\left(\lambda_{3}^{*}<\lambda<\lambda_{4}^{*}\right) \cup \cdots\right) \\
& =P\left(\lambda_{1}^{*}<\lambda<\lambda_{2}^{*}\right)+P\left(\lambda_{3}^{*}<\lambda<\lambda_{4}^{*}\right) \cdots
\end{aligned}
$$

[Figure 14 about here.]

Figures 14(a) and 14(b) compares Monte Carlo simulations against the multiroot finding method, for calculating the probability of failure for a body subjected to 2 cycoidal pulses, under the assumption that the values of $\lambda$ following the uniform distribution. It can be seen that there is an excellent match between the two methods and the same can be observed for any underlying assumed distribution of $\lambda$ or assumed multi-cycle cycloidal input. Using the multiroot method suggested, the probability of the body failing or surviving can be easily and quickly determined without the need for further dynamic simulations once the roots have been calculated, even if the assumed distribution of $\lambda$ changes. Hence, Monte Carlo simulations and the associated computational cost may be avoided. 
[Figure 15 about here.]

Comparing the single pulses against the multiple pulses, it can be noticed that similar trends in terms of the probabilities of failure can be observed. Despite the fact that equations 8 and 9 are not applicable anymore, the probability of failure still depends on the assumed distribution of $\lambda$ as can be seen in Figures 15(a) and 15(b) where a uniform and Truncated Gaussian distribution of $\lambda$ are compared when the body is subjected to three cosinoidal pulses.

\section{Stability of Rocking Bodies Subjected to Simu- lated Earthquakes}

The response of a rocking block subjected to cycloidal pulses was examined in sections Deterministic effect of $\lambda$ on the stability of the body and Probabilistic models for the effect of $\lambda$ on the stability of the body. However, ground motions cannot in generally be approximated with idealized pulses as the ones examined so far. Hence, there is a need to study the effect of the propagation of the uncertainty of $\lambda$ on the stability of rocking bodies subjected to earthquakes. For the purpose of this study, artificially generated earthquakes are used. To this end the Spectral Representation method defined in (Shinozuka and Deodatis, 1991) is used together with the Kanai-Tajimi, Clough-Penizen spectrum (Clough and Penzien, 1975):

$$
G(\omega)=S_{0} \frac{\left(1+4 \xi_{g}^{2}\left(\frac{\omega}{\omega_{g}}\right)^{2}\right)}{\left(1-\left(\frac{\omega}{\omega_{g}}\right)^{2}\right)^{2}+4 \xi_{g}^{2}\left(\frac{\omega}{\omega_{g}}\right)^{2}} \frac{\left(\frac{\omega}{\omega_{f}}\right)^{4}}{\left(1-\left(\frac{\omega}{\omega_{f}}\right)^{2}\right)^{2}+4 \xi_{f}^{2}\left(\frac{\omega}{\omega_{f}}\right)^{2}}
$$

where $S_{0}$ denotes the ground intensity, $\omega_{g}$ and $\xi_{g}$ are the natural frequency and damping ratio of the ground, and $\omega_{f}$ and $\xi_{f}$ are positive parameters related to a high-pass filter that ensures finite velocities when $\omega=0$. To introduce the non-stationarity of the process, a time modulation function $\varphi(t)$ developed by Jennings et al. (1968) Jennings et al. (1968) is applied.

$$
\varphi(t)=\left\{\begin{array}{ll}
t / t_{1} & t<t_{1} \\
1 & t_{1} \leq t \leq t_{2} \\
e^{-\beta\left(t-t_{2}\right)} & t>t_{2}
\end{array}\right\}
$$

To generate the records the values used in equations 19 and 20 equation are defined as $\omega_{g}=15 \mathrm{rad} / \mathrm{sec}, \xi_{g}=0.6, \omega_{f}=1.5 \mathrm{rad} / \mathrm{sec}, \xi_{f}=0.6, t_{1}=1, t_{2}=9$ and $\beta=0.2$, corresponding to a firm soil.

[Figure 16 about here.]

To quantify the risk of failure for the body subjected to the generated time histories, the probability of failure is calculated after varying $\sqrt{S_{0}}$ as a measure 
of intensity and generating a suitable number of records. For this end, 10000 earthquakes are generated for $S_{0}=1$ and are later uniformly scaled by multiplying the occuring amplitudes with $\sqrt{S_{0}}$. The probability is calculated using a Monte Carlo analysis as the sum of records for which failure occurs over the total number of records. Depending on the assumed value of $\lambda$ a different curve is produced as shown in Figure 16.

As expected, the probability of failure, for a given $\lambda$, increases as the value of the intensity measure, $S_{0}$, increases. However, it should be noted that the probability of failure also increases for a given of $S_{0}$ when the assumed value of $\lambda$ is increased. This indicates once again that the use of zero values for $\lambda$ may potentially underestimate the probability of failure of the body, a conclusion compatible to the results seen in earlier sections of this paper on cycloidal pulses.

\section{Conclusions}

This paper discusses the assumptions involved in modelling the loss of energy during impact in the $I P M$, even if sliding, uplift, ground deformability and the three dimensional nature of the response can indeed be neglected. It is shown that the location of the vertical impulse during impact could be applied at any point between the post-impact rocking corner of the body and the midpoint of the base, without violating any of the assumptions of the model. The effect of the vertical impulse acting at a distance $\lambda$ (for $\lambda$ between zero and one) from the future rocking corner was investigated first in terms of the stability of a rocking body subjected to single cycloidal pulses. It was illustrated that the assumption for the value of $\lambda$ significantly affects the stability of rocking bodies and that the assumption of $\lambda=0$, common in the literature, is the least conservative assumption compatible with the assumptions of the IPM. A property of the dynamic system was highlighted, where increasing the value of $\lambda$ for a point in the stability diagram results in expanding the area of failure with impact monotonically.

Defining $\lambda$ as random variable, the probability of failure of the body is quantified. A probabilistic method based on the dynamic behavior of the system, defined in equation 9 , was introduced to calculate efficiently the probability of failure of a rocking body. The method was verified against Monte-Carlo Simulations. Using this method, it was shown that there may be points in the stability diagram whose probability of failure is close to $50 \%$ and hence the prediction of the model on whether the body will survive or fail is associated with a large uncertainty. The size and location of this uncertain region was shown to be dependent on the assumed distribution of $\lambda$.

The previous were then extended in terms of stability of a body subjected to multiple pulses. A method was developed to calculate the corresponding probability of failure of the body by extending the probabilistic method presented for the case of multiple transitions between survival and failure. It was again shown that the uncertainty in $\lambda$ has a significant effect in the stability of a rocking body. The final example illustrated the dependence of fragility curves for 
rocking bodies subjected to artificially generated earthquakes on the assumed value of $\lambda$, where again it was shown that the assumption of $\lambda=0$ may result in underestimating the probability of failure for the body.

This paper shows the need for taking into account the uncertainty related to the assumption of how energy is lost during impacts, as expressed through the range of allowable values of $\lambda$ that are compatible with the assumptions of the IPM. In order to reduce this uncertainty one could conduct experiments to further characterize a suitable distribution of $\lambda$, or mechanically modify the body to make the assumption of $\lambda$ being zero more probable. This however would still not guarantee that $\lambda$ would be fixed for that body and that for example would not depend on the value of the pre-impact velocities, an assumption that was retained in this paper. Finally, one would still need to take into account that there are several cases where the omitted phenomena of sliding, uplift, deformability and three dimensional nature of the response would affect the behaviour of the body by allowing for dynamic patterns that cannot be approximated from the IPM, through the use of any value of $\lambda$.

The latter assumptions can be relaxed with suitable models, as for example the spring models proposed in (Chatzis and Smyth, 2012b) and (Chatzis and Smyth, 2012a). Although such models do require the suitable identification of the parameters of the springs and dampers used to model the behaviour of the body, it should be noted that those could potentially be identified using system identification methods or through material models suitable for each application. In parallel the models would also be capable of taking into account phenomena omitted by the IPM. Hence, use of such models may be a viable alternative to the IPM and its inherent uncertainty illustrated in this paper.

\section{Acknowledgements}

The first two authors would like to acknowledge the financial support of the EC, FP7-PEOPLE-2013, Marie Curie, Career Integration Grant, RERCSGM, project number 618359 . The third author would like to acknowledge the NSF grant CMMI 1200859.

\section{References}

Chatterjee, A. and Ruina, A. (1998), 'A new algebraic rigid-body collision law based on impulse space considerations', Journal of Applied Mechanics 65(4), 939-951.

Chatzis, M. N. and Smyth, A. W. (2012a), 'Modeling of the 3d rocking problem', International Journal of Non-Linear Mechanics 47, 85-98.

Chatzis, M. N. and Smyth, A. W. (2012b), 'Robust modeling of the rocking problem', Journal of Engineering Mechanics 3, 247-262.

URL: doi:10.1061/(ASCE)EM.1943-7889.0000329 
Clough, R. W. and Penzien, J. (1975), Dynamics of Structures, Mcgraw-Hill.

Dimitrakopoulos, E. G. and DeJong, M. J. (2012), 'Revisiting the rocking block: closed-form solutions and similarity laws', Proceedings of the Royal Society of London A: Mathematical, Physical and Engineering Sciences .

URL: http://rspa.royalsocietypublishing.org/content/early/2012/04/03/rspa.2012.0026

Dormand, J. and Prince, P. (1980), 'A family of embedded runge-kutta formulae', Journal of Computational and Applied Mathematics 6(1), 19 - 26.

URL: http://www.sciencedirect.com/science/article/pii/0771050X80900133

ElGawady, M. A., Ma, Q., Butterworth, J. W. and Ingham, J. (2011), 'Effects of interface material on the performance of free rocking blocks', Earthquake Engineering 83 Structural Dynamics 40(4), 375-392.

URL: http://dx.doi.org/10.1002/eqe.1025

Housner, G. W. (1963), 'The behavior of inverted pendulum structures during earthquakes', Bulletin of the Seismological Society of America 53, 403-417.

Ishiyama, Y. (1982), 'Motion of rigid bodies and criteria for overturning by earthquake excitations', Journal of Earthquake Engineering and Structural Dynamics 10(10), 635-650.

Jennings, P., Housner, G. W. and Tsai, N. C. (1968), Simulated Earthquake Motions.

Koh, A., Spanos, P. D. and Roesset, J. (1986), 'Harmonic rocking of rigid block on flexible foundation', Journal of Engineering Mechanics 112(11).

Mathworks (n.d.), 'Matlab's ode event location', http://uk.mathworks.com/ help/matlab/math/ode-event-location.html. Accessed: 2016-08-28.

Palmeri, A. and Makris, N. (2008), 'Response analysis of rigid structures rocking on viscoelastic foundation', Earthquake Engineering and Structural Dynamics 37, 1039-1063.

Plaut, R., Fielder, W. and Virgin, L. (1996), 'Fractal behavior of an asymmetric rigid block overturning due to harmonic motion of a tilted foundation', Chaos, Solitons $\&$ Fractals $\mathbf{7}(2)$, 177-196.

Psycharis, I. N. and Jennings, P. C. (1983), 'Rocking of slender rigid bodies allowed to uplift', Earthquake Eng Struct Dyn 11(11), 57-76.

Robert, C. (1995), 'Simulation of truncated normal random variables', Statistics and Computing 5, 121-125.

Shampine, L. F. and Gordon, M. K. (1975), Computer solution of ordinary differential equations: the initial value problem, WH Freeman San Francisco.

Shenton, H. and Jones, N. (1991), 'Base excitation of rigid bodies. 1: Formulation', Journal of Engineering Mechanics 117, 2286-2306. 
Shinozuka, M. and Deodatis, G. (1991), 'Simulation of stochastic processes by spectral representation', American Society of Mechanical Engineering 44(4), 191-204.

Virgin, L., Fielder, W. and Plaut, R. (1996), 'Transient motion and overturning of a rocking block on a seesawing foundation', Journal of Sound and Vibration 191(1), $177-187$.

URL: http://www.sciencedirect.com/science/article/pii/S0022460X96901145

Voyagaki, E., Psycharis, I. and Mylonakis, G. (2014), 'Complex response of a rocking block to a full-cycle pulse', Journal of Engineering Mechanics 140(6), 04014024.

URL: http://dx.doi.org/10.1061/(ASCE)EM.1943-7889.0000712

Yim, C.-S., Chopra, A. K. and Penzien, J. (1980), 'Rocking response of rigid blocks to earthquakes', Earthquake Engineering $\&$ Structural Dynamics 8(6), 565-587.

URL: http://dx.doi.org/10.1002/eqe.4290080606

Zhang, H., Brogliato, B. and Liu, C. (2012), 'Study of the planar rockingblock dynamics with coulomb friction: Critical kinetic angles', Journal of Computational and Nonlinear Dynamics $\mathbf{8}^{\mathbf{6}}(2)$.

Zhang, J. and Makris, N. (2001), 'Rocking response of free-standing blocks under cycloidal pulses.', Journal of Engineering Mechanics 127(5), 473-483.

Zulli, D., Contento, A. and Egidio, A. D. (2012), '3d model of rigid block with a rectangular base subject to pulse-type excitation', International Journal of Non-Linear Mechanics 47(6), 679 - 687.

URL: http://www.sciencedirect.com/science/article/pii/S0020746211002563 

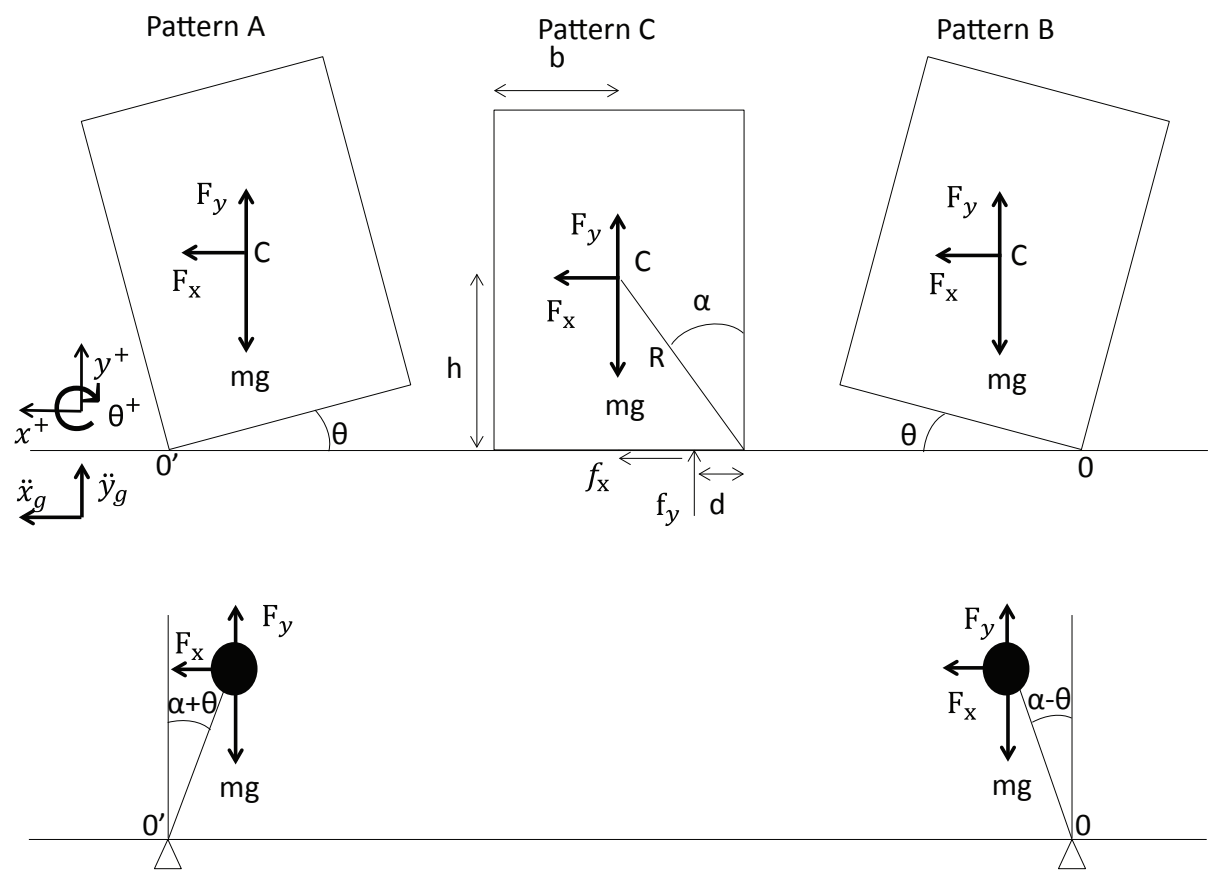

Figure 1 - Inverted Pendulum model defined by Housner Housner (1963) for a free-standing rocking block. 


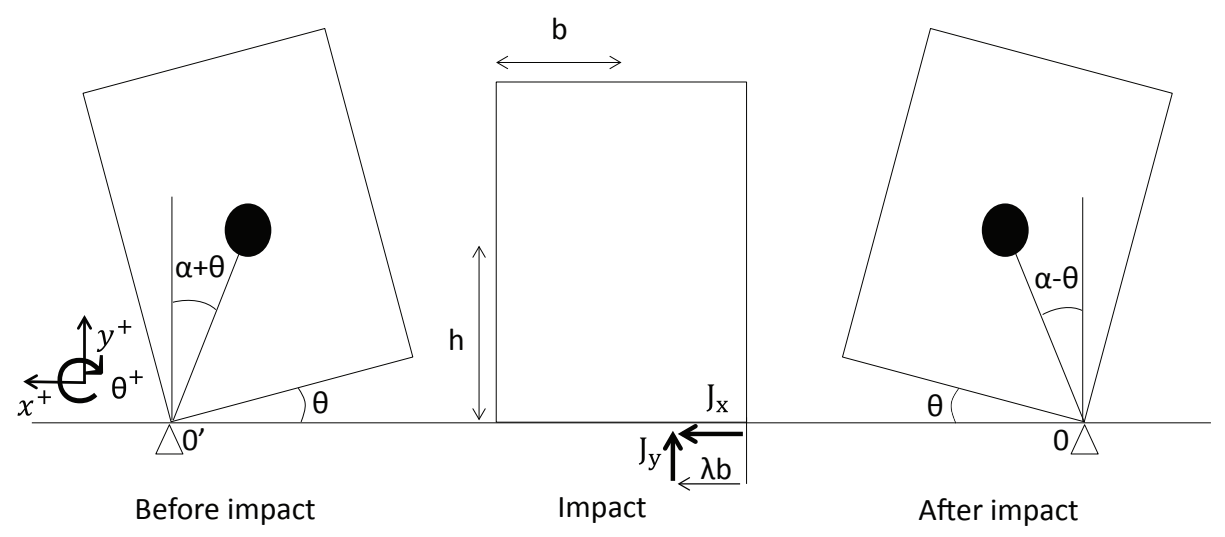

Figure 2 - It is assumed, with any loss of generality, that the impact occurs switching from Pattern A to B. At impact the body experiences an horizontal, $J_{x}$ and vertical, $J_{y}$, impulses at $\lambda$. 


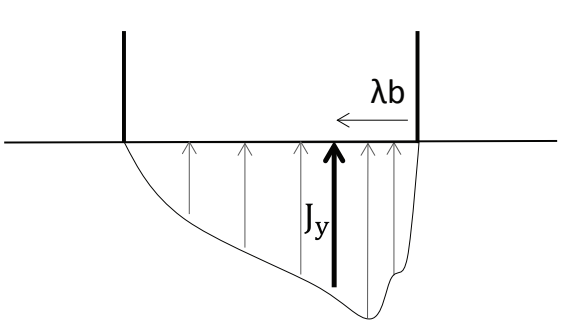

(a) Physically possible

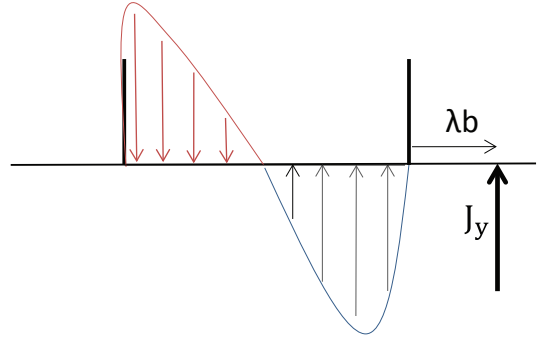

(b) Non-physically possible

Figure 3 -a) One of the infinitely many admissible distributions to describe the vertical impulse during impact. $J_{y}$ is the relative vertical impulse. b) The red distribution corresponds to tensile stresses which is a violation of the non-tensile assumption. 


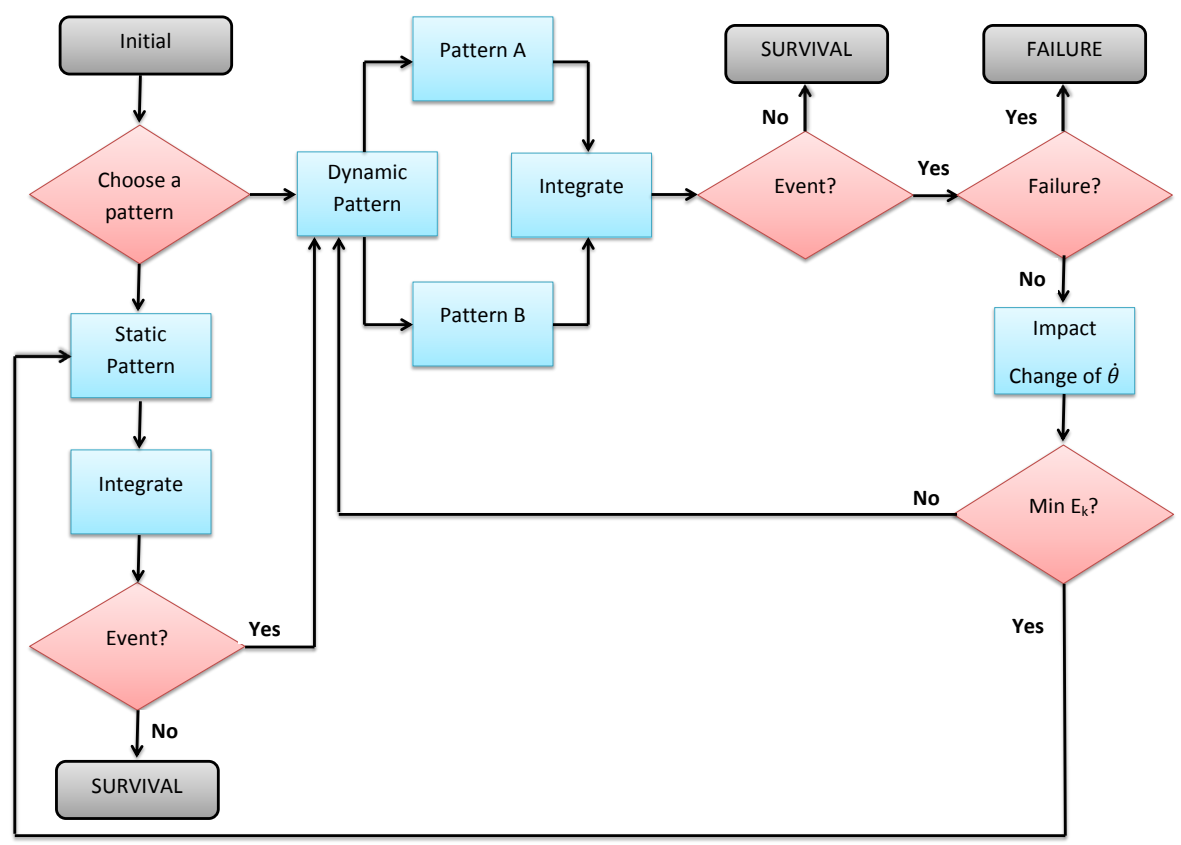

Figure 4 - Schematic explanation of the time integration scheme used. 


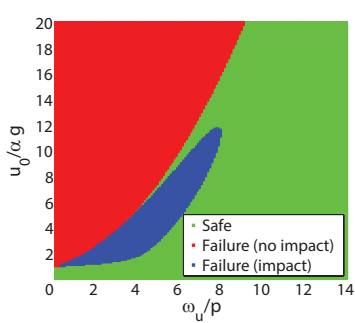

(a) $\lambda=0$, Sinusoidal

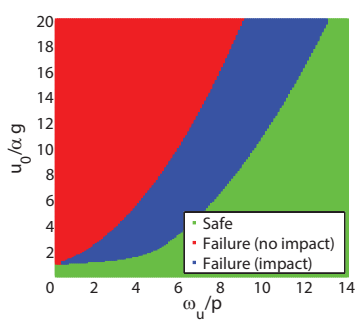

(d) $\lambda=1$, Sinusoidal

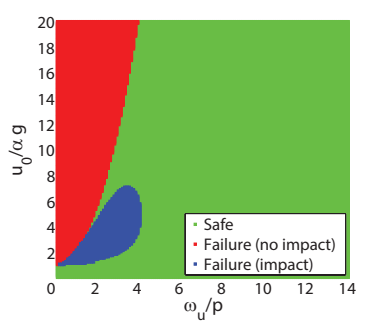

(b) $\lambda=0$, Cosinoidal

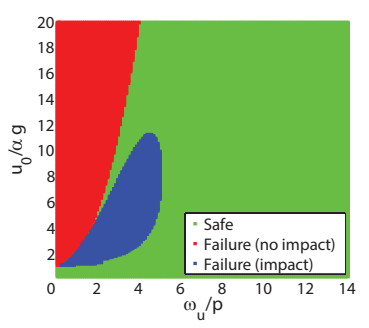

(e) $\lambda=1$, Cosinoidal

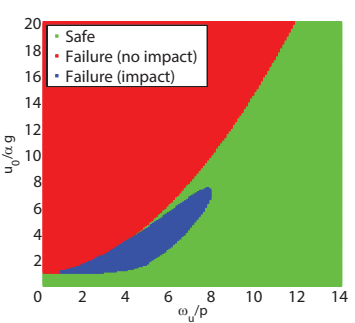

(c) $\lambda=0$, Square

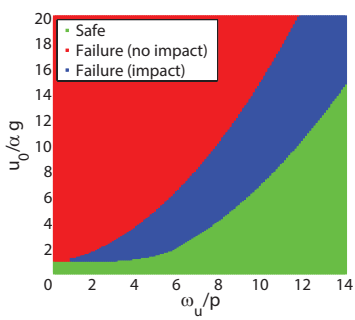

(f) $\lambda=1$, Square

Figure 5 - Overturning Acceleration Spectrum of Free standing block subjected to a single sinusoidal (a, c), cosinoidal (b, d) and square (c, f) acceleration pulse with frequency $\omega_{u}, \mathrm{p}=2.1401$ and $\alpha=0.2501$. 


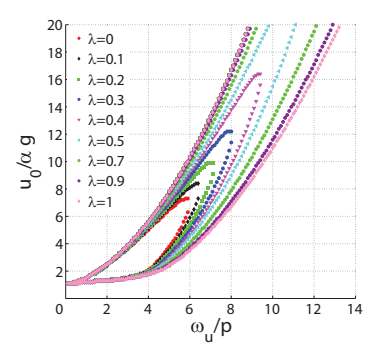

(a) Sinusoidal $\alpha=0.3218$

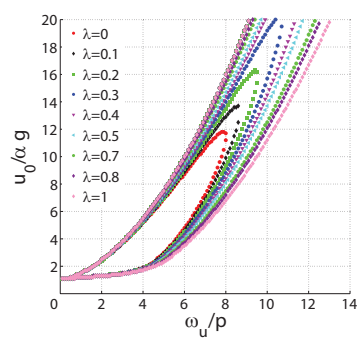

(d) Sinusoidal $\alpha=0.2501$

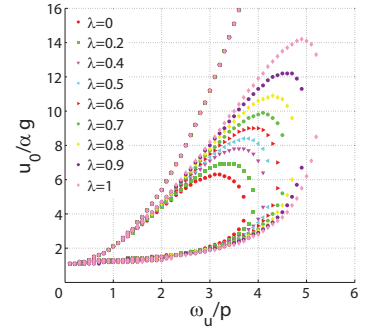

(b) Cosinoidal $\alpha=0.3218$

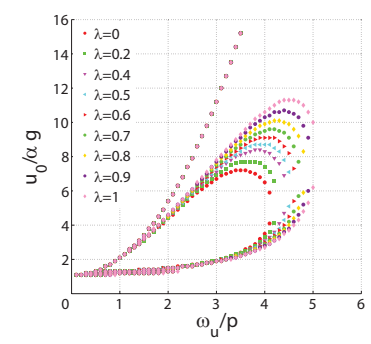

(e) Cosinoidal $\alpha=0.2501$

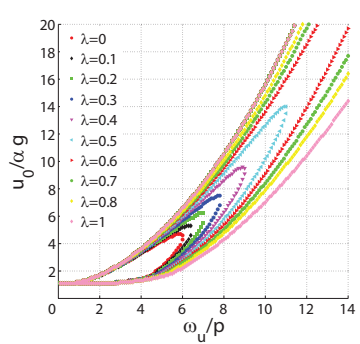

(c) Square $\alpha=0.3218$

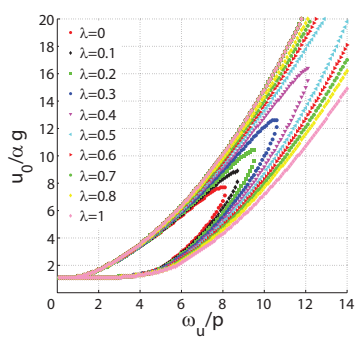

(f) Square $\alpha=0.2501$

Figure 6 - Boundary regions which separate the area of failure with impact from the failure without impact and survival. The block is subjected to a single sinusoidal (a, d) cosinoidal (b, e) and (c, f) square acceleration pulse with a constant $p=2.1401$ for $\alpha=0.3218$ and for $\alpha=0.2501$. 


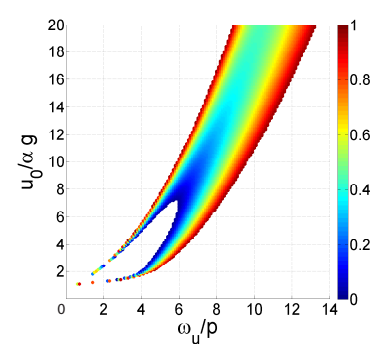

(a) Sinusoidal $\alpha=0.3218$

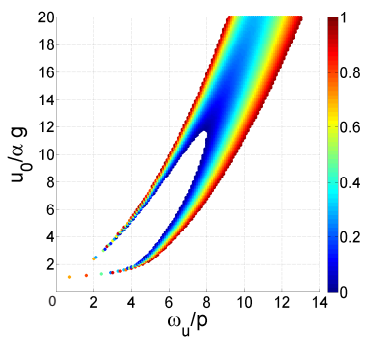

(d) Sinusoidal $\alpha=0.2501$

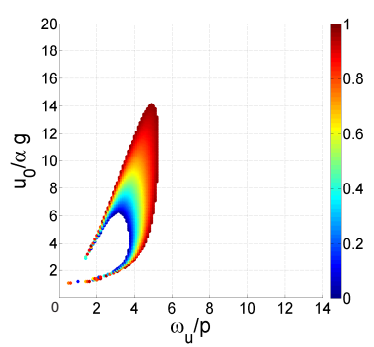

(b) Cosinoidal $\alpha=0.3218$

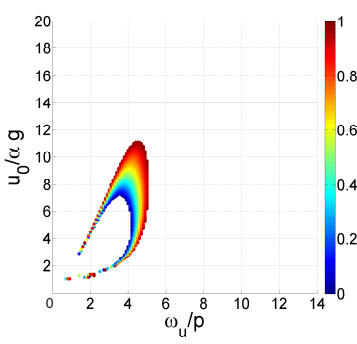

(e) Cosinoidal $\alpha=0.2501$

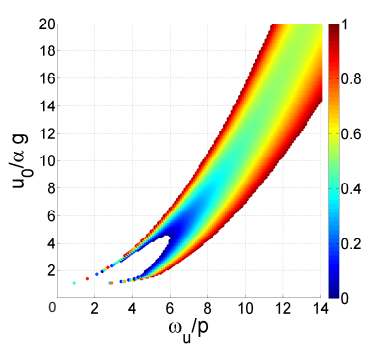

(c) Square $\alpha=0.3218$

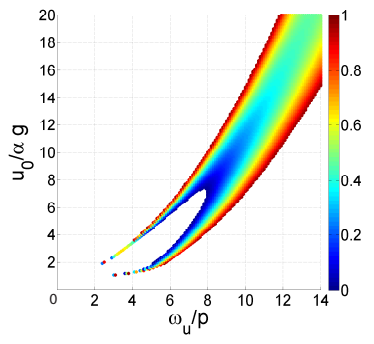

(f) Square $\alpha=0.2501$

Figure 7 - Values of $\lambda^{*}$ of a free standing block with a constant frequency parameter $p=2.1401$ subjected to a single sinusoidal for a) $\alpha=0.3218$ and d) $\alpha=0.2501$, to a cosinoidal for b) $\alpha=0.3218$ and e) $\alpha=0.2501$ and to a square pulse for c) $\alpha=0.2501$ and f) $\alpha=0.1244$. 


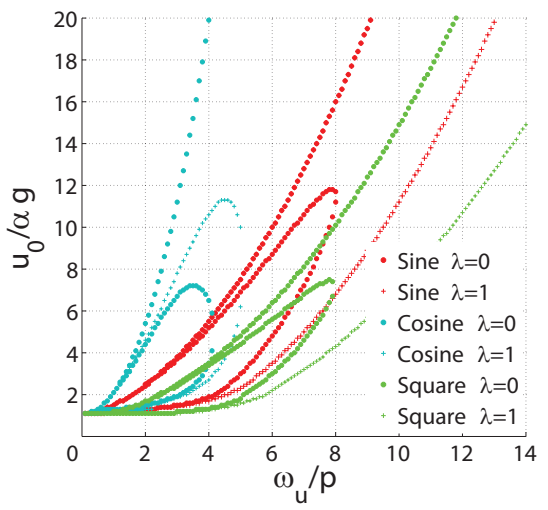

Figure 8 - Overturning Acceleration Spectrum for a single sinusoidal, cosinoidal and square pulse for values of $\lambda=0$ and $\lambda=1$ subjected to a rigid body with $p=2.1401$ and $\alpha=0.2501$. 


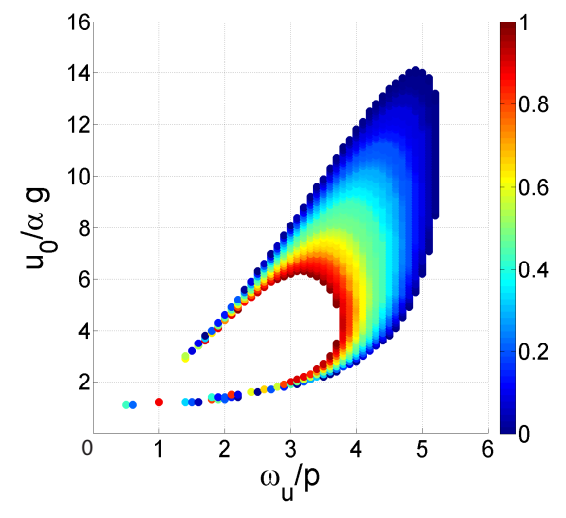

(a) Monte Carlo Simulation

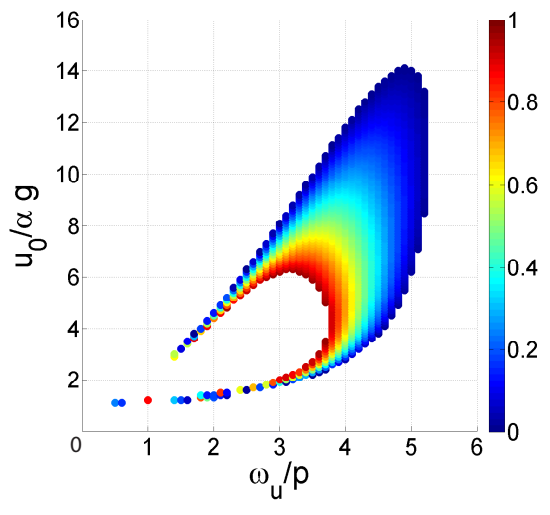

(b) Probabilistic approach

Figure 9 - Probability of Failure for one cosinoidal pulse using (a) Monte Carlo Simulation and (b) the Probabilistic approach for a body with $p=2.1401$ and $\alpha=0.3218$. 


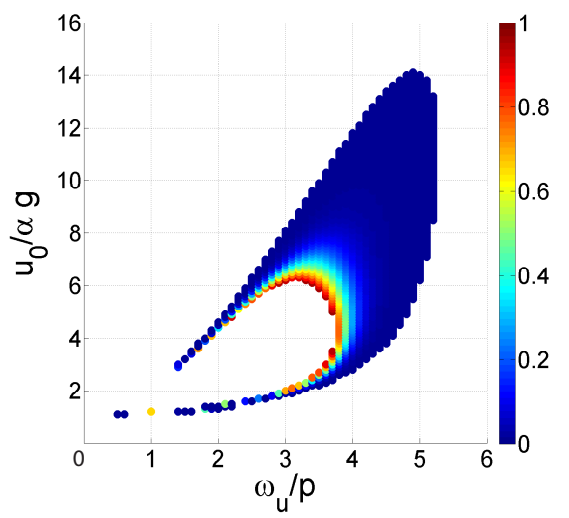

(a) $\mu=0.2$ and $\sigma=0.15$

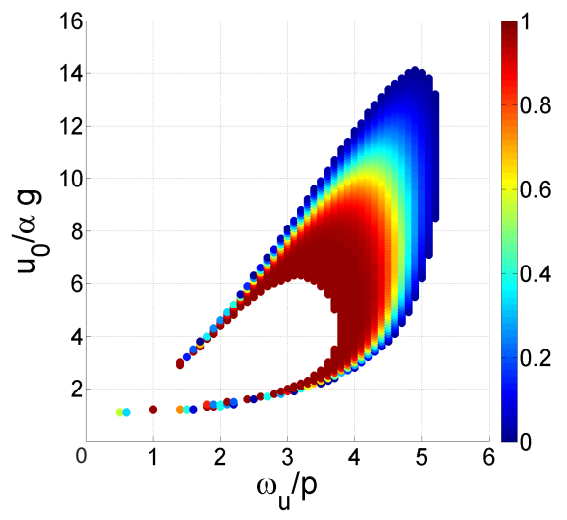

(c) $\mu=0.8$ and $\sigma=0.15$

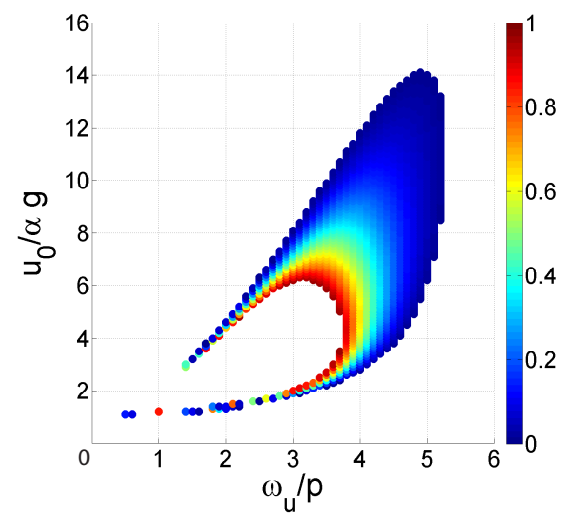

(b) $\mu=0.2$ and $\sigma=0.5$

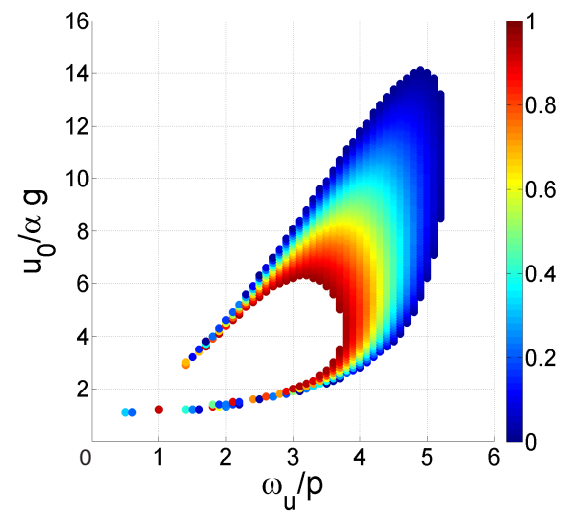

(d) $\mu=0.8$ and $\sigma=0.5$

Figure 10 - Probability of Failure for one single cosinoidal pulse using the truncated Gaussian distribution with a) $\mu=0.2$ and $\sigma=0.15$, b) $\mu=0.2$ and $\sigma=0.5$, c) $\mu=0.8$ and $\sigma=0.15$ and d) $\mu=0.8$ and $\sigma=0.5$ for a body with $p=2.1401$ and $\alpha=0.3218$. 


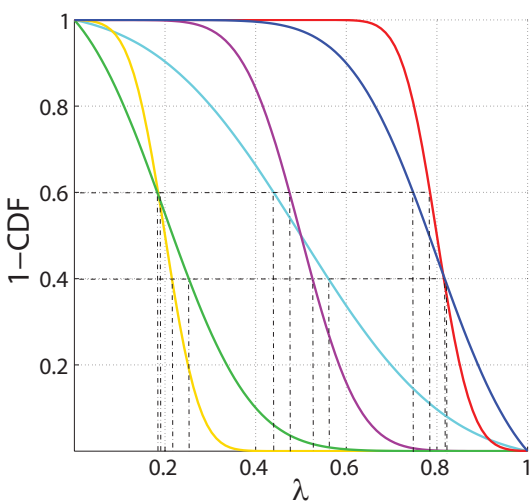

(a) $C D F$

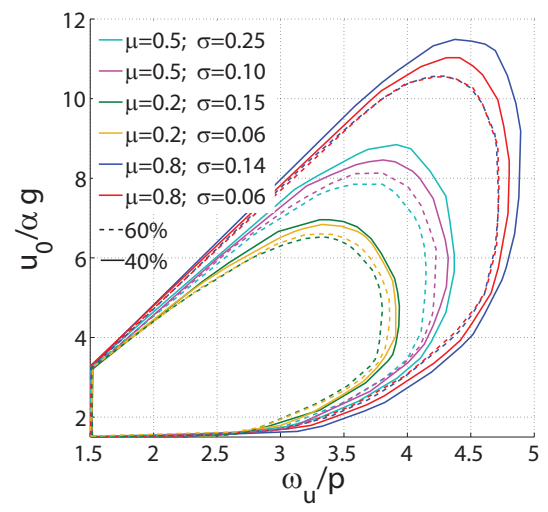

(b) Boundaries of probability of $40 \%-60 \%$

Figure $11-C D F$ (a) and $\lambda^{*}$ boundaries for probabilities between $40-60 \%$ (b) for a rigid body subjected to a single cosinoidal pulse for a body with $p=2.1401$ and $\alpha=0.3218$. 


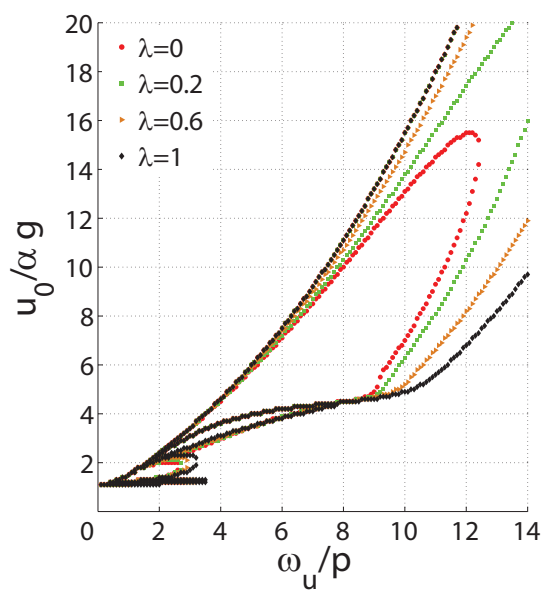

(a) Sine Pulse

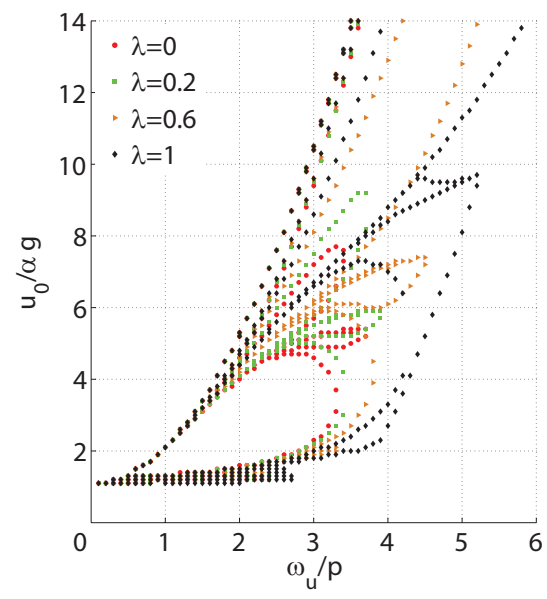

(b) Cosine Pulse

Figure 12 - Boundaries region which separate the area of failure with impact from the failure without impact and survival for a body with $p=2.1401$ and $\alpha=0.3218$. The block is subjected to a) double sinusoidal pulse and b) double cosinoidal pulse. 


\begin{tabular}{|c|c|c|c|c|c|c|}
\hline$F$ & $\mathrm{~S}$ & $\mathrm{~F}$ & $\mathrm{~s}$ & $\ldots$ & $\ldots$ & $\ldots$ \\
\hline & & 2 & & & $\left.\lambda_{n-1}\right\rangle$ & \\
\hline$S$ & $\mathrm{~F}$ & S & $\mathrm{F}$ & $\ldots$ & $\ldots$ & $\ldots$ \\
\hline & & & & & & \\
\hline
\end{tabular}

Figure 13 - Graph explaining the two possible cases that can happen using the multiroot finding method. 


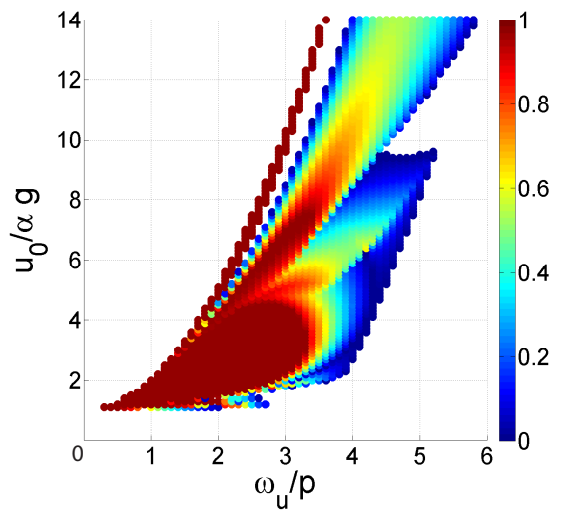

(a) Monte Carlo simulation

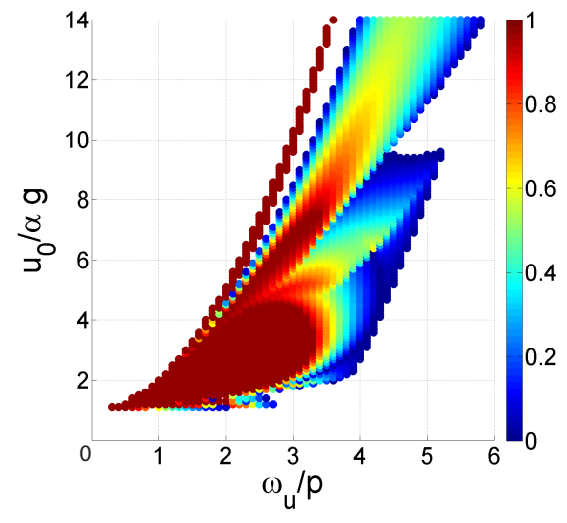

(b) Multiroots finding method

Figure 14 - Probabilistic approach for the Uniform Distribution for two cosinoidal pulses using a) Monte Carlo simulation and b) Multiroots finding method for a body with $p=2.1401$ and $\alpha=0.3218$. 


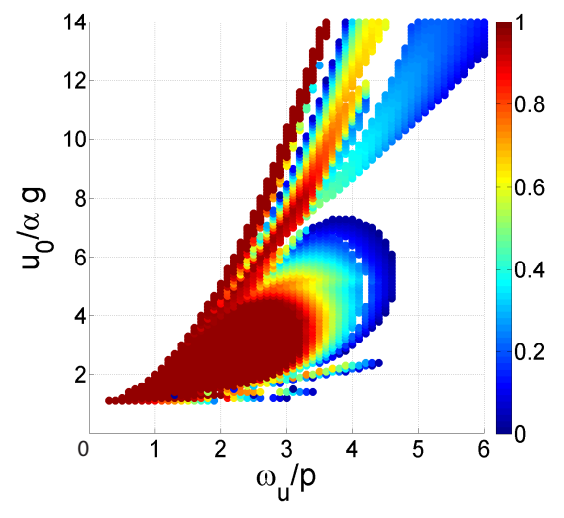

(a) Uniform Distribution

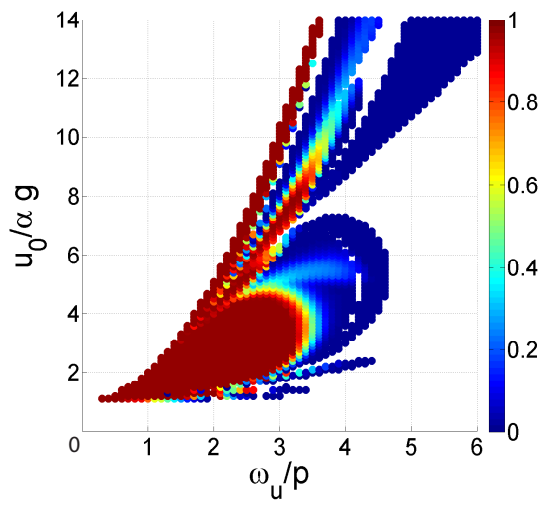

(b) Truncated Distribution

Figure 15 - Probabilistic approach for the Uniform Distribution for three cosinoidal pulses using the multiroot finding method using a) the uniform distribution and b) the Truncated Gaussian with $\mu=0.2, \sigma=0.15$ for a body with $p=2.1401$ and $\alpha=0.3218$. 


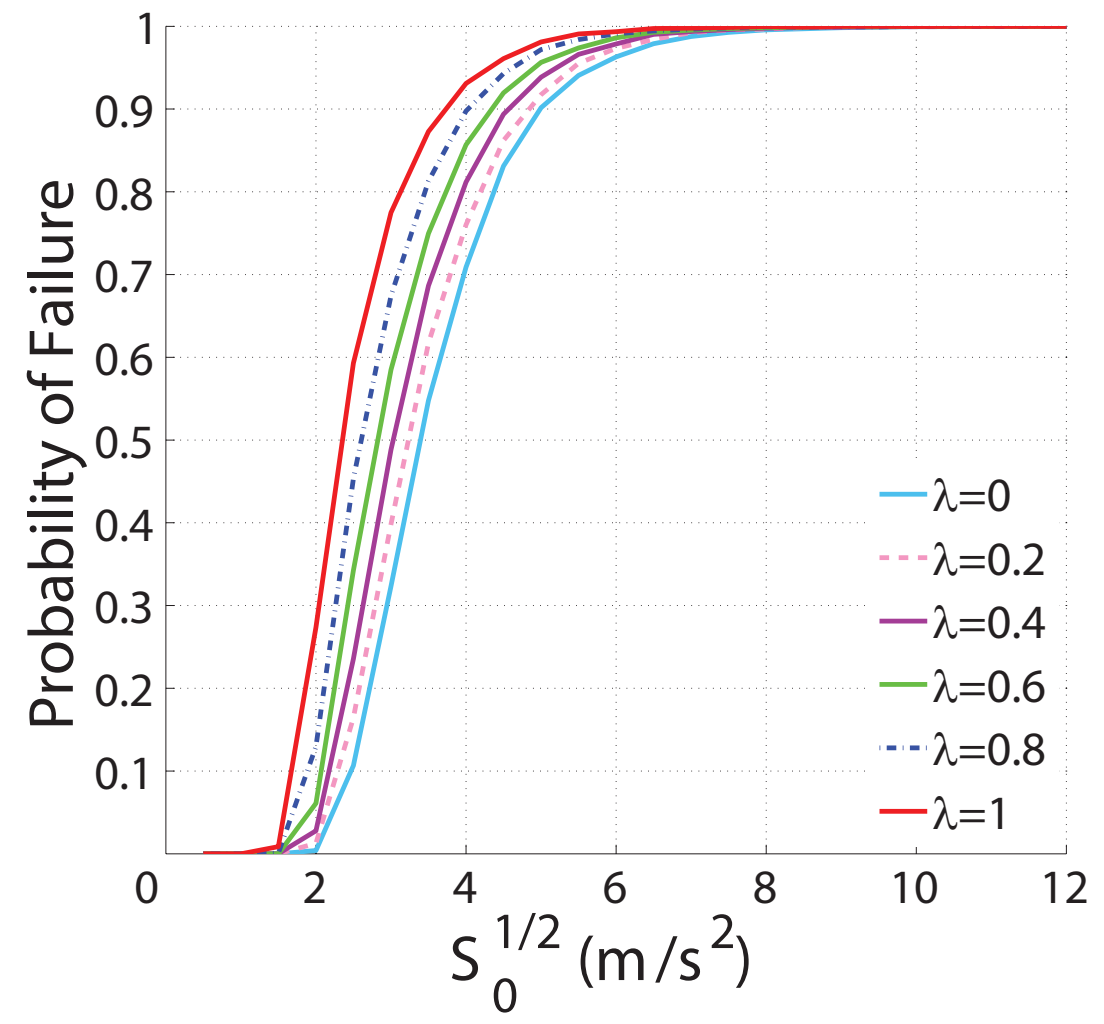

Figure 16 - Probability of failure for different values of $\sqrt{S_{0}}$ using 10.000 artificial earthquakes for different values of $\lambda$ for a body with $p=2.1401$ and $\alpha=0.3218$. 


\section{${ }_{691}$ List of Tables}

$\lambda^{*}$ values for $40-60 \%$ for truncated distributions of different $\mu$ and $\sigma \ldots \ldots \ldots \ldots \ldots \ldots \ldots \ldots \ldots \ldots \ldots$ 
Table $1-\lambda^{*}$ values for $40-60 \%$ for truncated distributions of different $\mu$ and $\sigma$.

\begin{tabular}{|c|c|c|c|}
\hline$\mu$ & $\sigma$ & $\lambda_{40}^{*}$ & $\lambda_{60}^{*}$ \\
\hline \multirow{2}{*}{$\mathbf{0 . 2}$} & 0.06 & 0.2254 & 0.1781 \\
\cline { 2 - 4 } & 0.15 & 0.2116 & 0.1326 \\
\hline \multirow{2}{*}{$\mathbf{0 . 5}$} & 0.1 & 0.5260 & 0.4260 \\
\cline { 2 - 4 } & 0.25 & 0.5743 & 0.4750 \\
\hline \multirow{2}{*}{$\mathbf{0 . 8}$} & 0.06 & 0.8051 & 0.7949 \\
\cline { 2 - 4 } & 0.14 & 0.8604 & 0.7836 \\
\hline
\end{tabular}

